\title{
ROAD EXTRACTION TECHNIQUES FROM REMOTE SENSING IMAGES: A REVIEW
}

\author{
I. Kahraman ${ }^{1}$, I. R. $\operatorname{Karas}^{1}$, A. E. Akay ${ }^{2}$ \\ ${ }^{1}$ Karabük University, Faculty of Engineering, 78050 Karabük, Turkey - \{idriskahraman, ismail.karas $\} @$ karabuk.edu.tr \\ ${ }^{2}$ Bursa Technical University, Faculty of Forestry, 16310 Bursa, Turkey - abdullah.akay@ btu.edu.tr
}

KEY WORDS: Remote Sensing Image, Classification, Road Extraction, Road Feature

\begin{abstract}
:
The importance of analysis high resolution satellite imagery plays an important research topic for geographical information analysis of cities. Geospatial data plays an important role in important issues such as governmental, industrial, research topics on traffic management, road monitoring, GNSS navigation, and map updating. In this study, road detection from satellite imagery methods are classified as classification-based, knowledge-based, mathematical morphology and dynamic programming. In the beginning, the road structures including feature and model are analyzed. Then, the advantages and disadvantages of road detection methods are evaluated and summarizez their accuracy and performance based on road detection principles. Therefore, in order to obtain remarkable results for road detection, it is better to use more than one method. In after days, performing a complex road extraction from a satellite image is still a necessary and important research topic.
\end{abstract}

\section{INTRODUCTION}

After release of enhencement in land observation satellites, many technologies applied to the remote sensing images with image processing developed rapidly. The main purpose of the RS implementations is to gather meaningful and semantic information and clarify interested targets to understand the whole image. Road network extraction from a RS image has many complex difficulties but it is worth to study. Roads are the fundamental part of transportation, traffic management, city planning, GPS navigation and map updating. Due to rapid changes on road network in urban transportation system, it is required to extract road network for instant road mapping. Various road detecting approachs have been presented for road network extraction from high resolution satellite images.

\section{ROAD MODELS AND FEATURES}

Road extraction from RS images, image characteristics of road features have difficulties according to spectral and spatial resolution, weather conditions, sensor type, light change and soil characteristics. Practically, extracting a road network based on structural model is very complex. Therefore, analysis of road characteristics and road modeling are very important. Geometric features, photogrammetric features, topological features, functionality and texture features are the main road features.

Different road sections in the images have different properties for road extraction from an image. The geometric properties of a path are directly related to the path shape. Photometric features are more representative of the gray level and color of the path. Topological and functional properties are relatively easy, but it is difficult to use these properties in real applications.

\section{ROAD EXTRACTION ALGORITHMS}

Due to numerious applications that applies diffirent road extraction methods, it is not easy to classify the techniques in detail. High number of research studies show that, most of the methods for extracting road segments consist of klowledgebased, classification-based, morphology-based and dynamic programming. These methods are briefly described in the following section

\subsection{Classification-Based Methods}

Methods for classification-based techniques generally use geometric properties, photometric properties, and texture properties for road sections. The accuracy for classification is unsatisfactory due to misclassification among road and road-like objects such as roads and building structures, parking lots, area blocks and water areas.

Labeled samples are trained for supervised classification methods. For the accuracy of these methods depend on selected features and labeled samples. In general, three types of supervised classification method is described as follows.

\subsubsection{Artificial Neural Networks Classification Method}

The first studies were based on spectral and contextual information of image pixels for direct classification of back propagation neural networks and advanced model. Tu-Ko (2003) presented a powerful approach to the extraction of the roadway major line, where the neural network is trained with edge and spectral features. The results can have good results throughout the system, even with many segments that are not roadside. The back propagation neural network method presented tested the network structure by experimenting with different parameters and obtaining the optimal input vector [5]. The process of determining optimal input parameters, network 
structure, and termination conditions in education is quite troublesome.

A method have been implemented with back-propagation network for road detection. As a first step, spectral information was used for road detection. After that, as an input texture parameters for gray level co-occurrence matrix (GLCM), contrast, energy, entropy, and homogeneity were calculated obtained from the input image. For the purpose of optimizing the system performance and evaluate the contribution of texture parameters to path determination, determinated texture parameters were associated with spectral information. The output road map is demonstrated in Figure 1. [6].

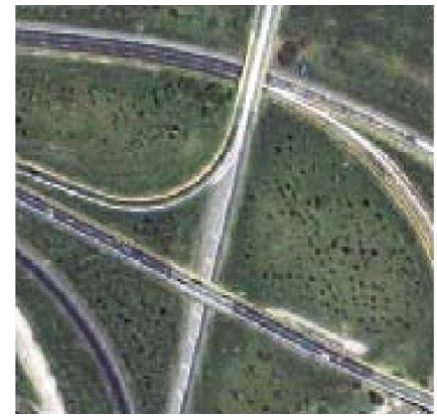

(a)

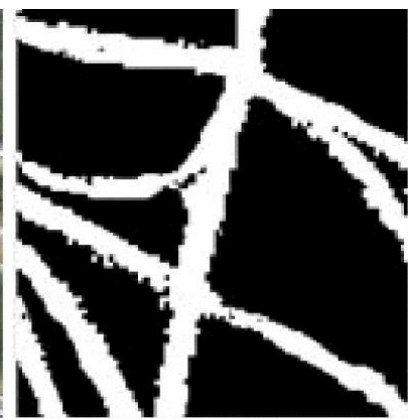

(b)
Figure 1. Road map, (a) input data, (b) output road map

Since, there are some disadvantages of the back propagation neural network methods such as; slow convergence, required more training samples, the performance is getting slow by increasing the number of classes, and it is easy to encounter over-fitting. Therefore, many newly enhenced neural network models is proposed to derive the road sections from RS images. For instance, radial floor nerve network function, fuzzy neural networks, spiking neural network, and hybrid neural network [4].

\subsubsection{Deep Learning Method}

Increasing the computing power with the GPU (Graphics Processing Unit) has increased the use of algorithms such as deep learning so growing data sets can be used for training. The most commonly applied method class for analyzing visual imagery in Deep Learning is convolutional neural network (CNN). In contrast to traditional machine learning methods where the features are chosen manually and extracted through instructed algorithms, representation learning is composed of methods that first feed pixel values or raw data into the system and allow it to automatically discover features or representations that can be used for detecting, distinguishing, and classifying patterns. Deep learning is very useful in learning and discovering fine and complex structures in high-dimensional data.

A method have been implemented propose a single patch-based Convolutional Neural Network (CNN) architecture for extraction of roads and buildings from high-resolution remote sensing data. This method does not consist any pre-processing stage. It is based on patch-based CNN, which consists of five convolution layers and replaces fully connected layers with GAP.Low-level features of roads and buildings (e.g., asymmetry and compactness) of adjacent regions are integrated with Convolutional Neural Network (CNN) features during the postprocessing stage to improve the performance. Figure 6 presents an example from Massachusetts data after applying convolutional network and low-level shape features. [16]. a

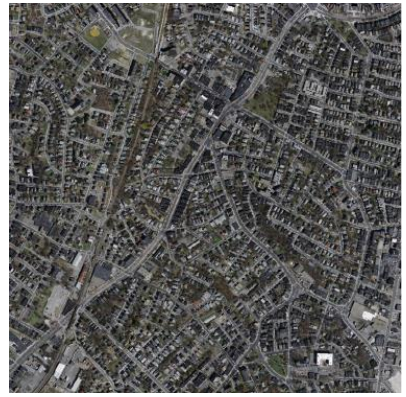

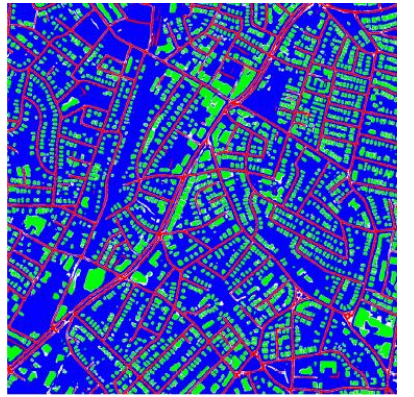

b
Figure 2. An example for CNN, (a) test image, (b) the result obtained by convololutional network and post-processing stage.

\subsubsection{Support Vector Machines Classification Method}

Risk minimization and generalization accuracy are the benefitial aspects for object detection using SVM classification methods in high resolution RS images. Hence, determining kernel functions, selection of domain and training samples constitute complexity using SVM methods.

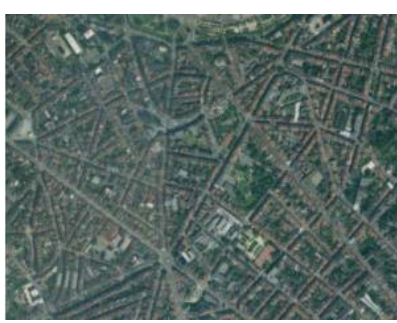

(a)

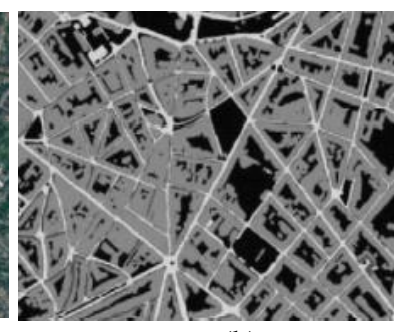

(b)
Figure 3. Extraction of road network. (a) Original image. (b) Extracted road network.

\subsubsection{Markov Random Field (MRF) Classification Methods}

A Markov Random Field (MRF) is a graphical model of a joint probability distribution. Due to spatial correlation between all pixels in an digital image, it can be anaylzed effectually by defining the conditional probability distribution function and texture statistical properties. MRF is widely used in areas such as image segmentation, edge detection, restoration and reconstraction. In can be seen from the studies and image below that relationship complexity between extracted objects in a satellite image makes it hard to model accurately by using just MRF model [15]. So, the hybrid model should be used to achieve remarkable results for complex object detectşon from satellite images. 


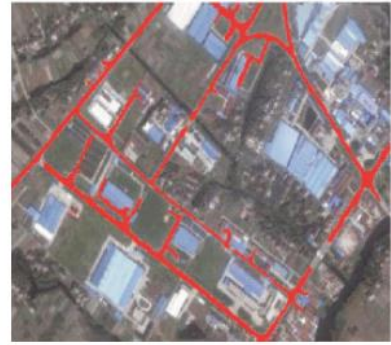

(a)

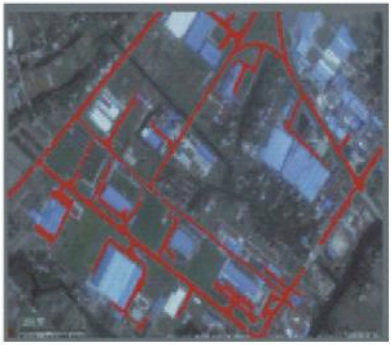

(b)
Figure 4. Two method for road extraction. (a) MRF-based detection. (b) SVM and FCM-based detection.

\subsection{Knowledge Based Methods}

It is hard to extract the roads from satellite images using only local spectrum and texture features. Since the structure of the road lane that makes identification of the eigenvector difficult, the data can not be entered directly into the classifier. For this reason, parameter models such as energy function can be used to work on the maximum value of the energy function. Common parameter models usually derive some structural elements from each other in relation to each other, and finally detect that it is specific enough to perform object detection [15]. Although the knowledge-based methods have been implemented to derive from RS images, it has some disadvantages such as oversubtraction, occlusions and shadowing.
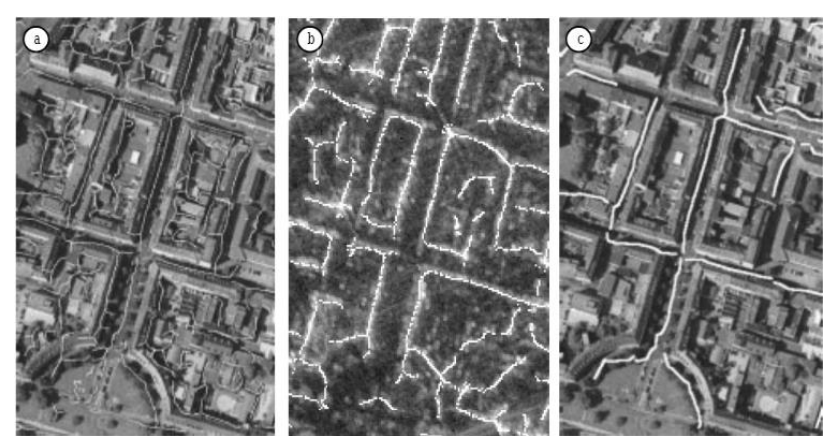

Figure 5: The segmented lines, i.e. road candidates, from the visual image (a) must be accompanied by parallel lines as hint for buildingsin the SAR image (b) to verify the road hypothesis (c) $[14]$

\subsection{Mathematical Morphology Methods}

Mathematical morphology takes a great deal of interest in academic areas, such as computer vision, image processing, pattern recognition, and other topics. Since 1980s, researchers proposed many methods based on mathematical morphology for road detection. Hence, image segmentation processes have been applied by mathematical morphology methods combined with different convenient methods.

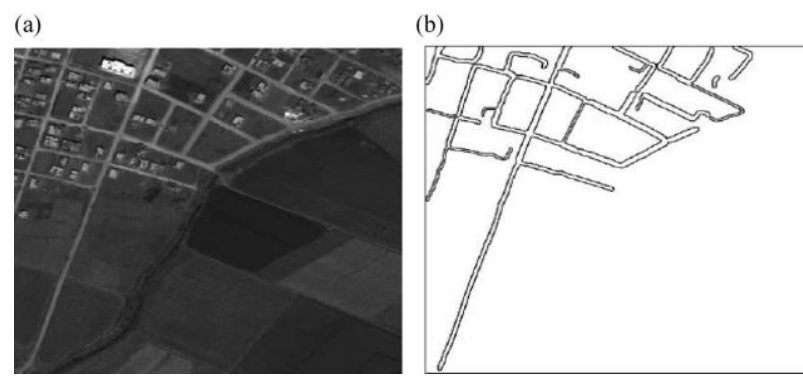

Figure 6. Road detection by Cem algorithm. (a) Ikonos image. (b) Road network detected.

Mathematical morphology methods reveal certain advantages that are commonly used in deriving from satellite images. The image segmentation outcome are considerably influenced by the selection of the structural elements by shape and size. Since the components of the construction elements, it is hard to use only the mathematical morphology method in order to obtain remarkable accuracy and significant extraction outcomes.

\subsection{Dynamic Programming And Grouping}

Dynamic programming is a metod that implements a mathematical method to perform the decision process. Ordinarily, the path must be given as a parameter model and expressed as a cost function. Dynamic programming is considered as a computation tool to specify the best path between the seed points. An algorithm for automatic road detecting from an satellite image with dynamic programming and Kalman filter for path monitoring is proposed. In spite of missing edge and occlusion of cars and bridges, it can detect roads simultaneously and in real time. But, there are particular limitations in this method for many assumptions as much as the previous information available in the monitoring operation. Movaghati et al. (2010) introduced a extracting model using the Extent Kalman filter and the Particle filter. These algorithms use a clustering algorithm without considering road barriers. This algorithm may follow all path intersections, but the result depends on the parameters that are set in the modüle [12].

(a)

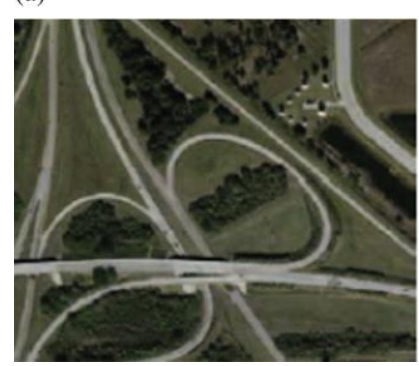

(b)

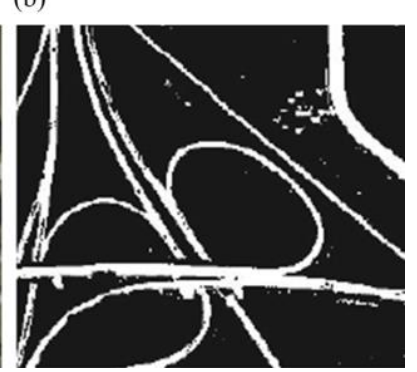

Figure 7. Road and road network extraction results. (a) Input image. (b) Output image.

\section{CONCLUSION}

As explained above, each algorithm has advantages and disadvantages according to the run time, accuracy and complexity. It can be detected roads from satellite images using only one algorithm. However, the accuracy will not be in remarkable accuracy, unless using combination of various methods. Additionally, The main problem for road detection from satellite images is to determine features of the road. A 
linear line is defined for road sections in most of the presented methods. After incrising advanced technology, it is easy to reach high resolution images. So many detailed road features will emerge including lots of noise. These roads should be defined fully. For this reason, many researchers concern about proper road model and how to detect roads in remarkable accuracy and quickly.

\section{ACKNOWLEGEMENT}

We would like to thank to the Karabuk University BAP unit for financial assistance.

\section{REFERENCES}

[1] Vosselman, G., Knecht, J., 1995. Road tracing by profile matching and Kalman filtering. In: Gruen, A., Baltsavias, E., Henricsson, O. (Eds.), Workshop on Automatic Extraction of Manmade Objects from Aerial and Space Images, Birkhauser, Berlin, pp. 265e274.

[2] Baumgartner, A., Steger, C., Mayer, H., et al., 1999. Automatic road extraction based on multi-scale, grouping, and context. Photogrammetric Engineering and Remote Sensing 65 (7), $777 \mathrm{e} 785$.

[3] Herumuti, D., Uchimura, K., Koutaki, G., et al., 2013. Urban road extraction based on hough transform and region growing. In: The 19th KoreaeJapan Joint Workshop on Frontiers of Computer Vision, Incheon, 2013.

[4] Simler, C., 2011. An improved road and building detector on VHR images. In: International Geoscience and Remote Sensing Symposium, Vancouver, 2011.

[5] Mokhtarzade, M., Valadanzoej, M.J., 2007. Road detection from high-resolution satellite imagery using artificial neural networks. International Journal of Applied Earth Observation and Geoinformation 9 (1), 32e40.

[6] Kirthika, A., Mookambiga, A., 2011. Automated road network extraction using artificial neural network. In: IEEEInternational Conference on Recent Trends in Information Technology, Chennai, 2011.

[7] Zhu, C., Shi, W., Pesaresi, M., et al., 2005. The recognition of road network from high-resolution satellite remotely sensed data using image morphological characteristics. International Journal of Remote Sensing 26 (24), 5493e5508.

[8] Wang, Y., Zheng, Q., 1998. Recognition of roads and bridges in SAR Images. Pattern Recognition 31 (7), 953e962.

[9] Hu, J., Razdan, A., Femiani, J.C., et al., 2007. Road network extraction and intersection detection from aerial images by tracking road footprints. IEEE Transactions on Geoscience and Remote Sensing 45 (12), 4144e4157.

[10] Zhang, D.B., 2007. Research on Interactive Road Extraction Method from High-resolution RS Image (PhD thesis). Xi'an Institute of Optics and Precision Mechanics, Chinese Academy of Scienee, Xi'an.

[11] Barzohar, M., Cooper, D.B., 1996. Automatic finding of main roads in aerial images by using geometric-stochastic models and estimation. IEEE Transactions on Pattern Analysis and Machine Intelligence 18 (7), 707e721.

[12] Movaghati, S., Moghaddamjoo, A., Tavakoli, A., 2010. Road extraction from satellite images using particle filtering and extended Kalman filtering. IEEE Transactions on Geoscience and Remote Sensing 48 (7), 2807e2817.

[13] Das, S., Mirnalinee, T.T., Varghese, K., 2011. Use of salient features for the design of a multistage framework to extract roads from high-resolution multispectral satellite images. IEEE Transactions on Geoscience and Remote Sensing 49 (10), $3906 \mathrm{e} 3931$.

[14] Tönjes, Ralf \& Growe, Stefan. (2002). Knowledge Based Road Extraction from Multisensor Imagery.

[15] Wixing Wang, Nan Yang, Yi Zhang, Fengping Wang, Ting Cao, Patrik Eklund, A review of road extraction from remote sensing images, Journal of Traffic and Transportation Engineering, Volume 3, Issue 3, June 2016, Pages 271-282

[16] Rasha Alshehhi, Prashanth Reddy Marpu, Wei Lee Woona, Mauro Dalla Mura, Simultaneous extraction of roads and buildings in remote sensing imagery with convolutional neural networks, ISPRS Journal of Photogrammetry and Remote Sensing, 130 (2017) 139-149

Revised August 2018 